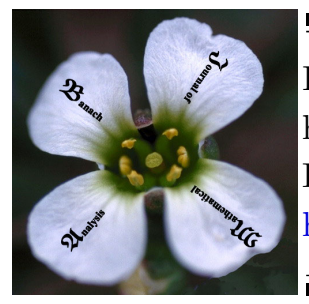

Banach J. Math. Anal. 9 (2015), no. 4, 359-378

http://doi.org/10.15352/bjma/09-4-17

ISSN: $1735-8787$ (electronic)

http://projecteuclid.org/bjma

\title{
LOCAL HERZ-TYPE HARDY SPACES WITH VARIABLE EXPONENT
}

\author{
HONGBIN WANG ${ }^{1 *}$ AND ZONGGUANG LIU ${ }^{2}$ \\ Communicated by T. Sugawa
}

\begin{abstract}
In this paper, we introduce a certain Herz-type Hardy space with variable exponent and establish the atom decomposition theorem for it. Using this decomposition, we obtain some boundedness on the Herz-type Hardy space with variable exponent for a class of pseudo-differential operators.
\end{abstract}

\section{INTRODUCTION}

Given an open set $\Omega \subset \mathbb{R}^{n}$, and a measurable function $p(\cdot): \Omega \rightarrow[1, \infty)$, $L^{p(\cdot)}(\Omega)$ denotes the set of measurable functions $f$ on $\Omega$ such that for some $\lambda>0$,

$$
\int_{\Omega}\left(\frac{|f(x)|}{\lambda}\right)^{p(x)} d x<\infty .
$$

This set becomes a Banach function space when equipped with the LuxemburgNakano norm

$$
\|f\|_{L^{p(\cdot)}(\Omega)}=\inf \left\{\lambda>0: \int_{\Omega}\left(\frac{|f(x)|}{\lambda}\right)^{p(x)} d x \leq 1\right\} .
$$

These spaces are referred to as variable Lebesgue spaces or, more simply, as variable $L^{p}$ spaces, since they generalized the standard $L^{p}$ spaces: if $p(x)=p$ is constant, then $L^{p(\cdot)}(\Omega)$ is isometrically isomorphic to $L^{p}(\Omega)$. The $L^{p}$ spaces with variable exponent are a special case of Musielak-Orlicz spaces.

Date: Received: Oct. 13, 2014; Revised: Jan. 8, 2015; Accepted: Mar. 1, 2015.

* Corresponding author.

2010 Mathematics Subject Classification. Primary 46E30; Secondary 42B30, 42B35.

Key words and phrases. Herz-type Hardy space, variable exponent, atom decomposition. 
The space $L_{\text {loc }}^{p(\cdot)}(\Omega)$ is defined by

$$
L_{\mathrm{loc}}^{p(\cdot)}(\Omega):=\left\{f: f \in L^{p(\cdot)}(E) \text { for all compact subsets } E \subset \Omega\right\} .
$$

Define $\mathcal{P}(\Omega)$ to be the set of $p(\cdot): \Omega \rightarrow[1, \infty)$ such that

$$
p^{-}=\operatorname{essinf}\{p(x): x \in \Omega\}>1, \quad p^{+}=\operatorname{ess} \sup \{p(x): x \in \Omega\}<\infty .
$$

Denote $p^{\prime}(x)=p(x) /(p(x)-1)$.

Let $f \in L_{\text {loc }}^{1}\left(\mathbb{R}^{n}\right)$, the Hardy-Littlewood maximal operator is defined by

$$
\mathcal{M} f(x)=\sup _{r>0} \frac{1}{\left|B_{r}(x)\right|} \int_{B_{r}(x)}|f(y)| d y
$$

where $B_{r}(x)=\left\{y \in \mathbb{R}^{n}:|x-y|<r\right\}$. There exist some sufficient conditions on $p(\cdot)$ such that the maximal operator $\mathcal{M}$ is bounded on $L^{p(\cdot)}\left(\mathbb{R}^{n}\right)$, see $[1,2]$. Let $\mathcal{B}\left(\mathbb{R}^{n}\right)$ be the set of $p(\cdot) \in \mathcal{P}\left(\mathbb{R}^{n}\right)$ such that $\mathcal{M}$ is bounded on $L^{p(\cdot)}\left(\mathbb{R}^{n}\right)$.

In variable $L^{p}$ spaces there are some important lemmas as follows.

Lemma 1.1. ([6]) Let $p(\cdot) \in \mathcal{P}\left(\mathbb{R}^{n}\right)$. If $f \in L^{p(\cdot)}\left(\mathbb{R}^{n}\right)$ and $g \in L^{p^{\prime}(\cdot)}\left(\mathbb{R}^{n}\right)$, then $f g$ is integrable on $\mathbb{R}^{n}$ and

$$
\int_{\mathbb{R}^{n}}|f(x) g(x)| d x \leq r_{p}\|f\|_{L^{p(\cdot)\left(\mathbb{R}^{n}\right)}}\|g\|_{L^{p^{\prime}(\cdot)\left(\mathbb{R}^{n}\right)}}
$$

where

$$
r_{p}=1+1 / p^{-}-1 / p^{+} .
$$

This inequality is named the generalized Hölder inequality with respect to the variable $L^{p}$ spaces.

Lemma 1.2. ([5]) Let $q(\cdot) \in \mathcal{B}\left(\mathbb{R}^{n}\right)$. Then there exists a positive constant $C$ such that for all balls $B$ in $\mathbb{R}^{n}$ and all measurable subsets $S \subset B$,

$$
\begin{gathered}
\frac{\left\|\chi_{B}\right\|_{L^{q(\cdot)}\left(\mathbb{R}^{n}\right)}}{\left\|\chi_{S}\right\|_{L^{q(\cdot)}\left(\mathbb{R}^{n}\right)}} \leq C \frac{|B|}{|S|}, \\
\frac{\left\|\chi_{S}\right\|_{L^{q(\cdot)}\left(\mathbb{R}^{n}\right)}}{\left\|\chi_{B}\right\|_{L^{q(\cdot)}\left(\mathbb{R}^{n}\right)}} \leq C\left(\frac{|S|}{|B|}\right)^{\delta_{1}}, \frac{\left\|\chi_{S}\right\|_{L^{q^{(}(\cdot)}\left(\mathbb{R}^{n}\right)}}{\left\|\chi_{B}\right\|_{L^{q^{\prime}(\cdot)}\left(\mathbb{R}^{n}\right)}} \leq C\left(\frac{|S|}{|B|}\right)^{\delta_{2}},
\end{gathered}
$$

where $0<\delta_{1}, \delta_{2}<1$ are constants.

Throughout this paper $\delta_{1}$ and $\delta_{2}$ are the same as in Lemma 1.2.

Lemma 1.3. ([5]) Suppose $q(\cdot) \in \mathcal{B}\left(\mathbb{R}^{n}\right)$. Then there exists a constant $C>0$ such that for all balls $B$ in $\mathbb{R}^{n}$,

$$
\frac{1}{|B|}\left\|\chi_{B}\right\|_{L^{q(\cdot)\left(\mathbb{R}^{n}\right)}}\left\|\chi_{B}\right\|_{L^{q^{(\cdot)}\left(\mathbb{R}^{n}\right)}} \leq C .
$$

Firstly we give the definition of the Herz spaces with variable exponent. Let $B_{k}=\left\{x \in \mathbb{R}^{n}:|x| \leq 2^{k}\right\}$ and $A_{k}=B_{k} \backslash B_{k-1}$ for $k \in \mathbb{Z}$. Denote $\mathbb{N}$ and $\mathbb{Z}_{+}$as the sets of all positive and non-negative integers, $\chi_{k}=\chi_{A_{k}}$ for $k \in \mathbb{Z}, \tilde{\chi}_{k}=\chi_{k}$ if $k \in \mathbb{N}$ and $\tilde{\chi}_{0}=\chi_{B_{0}}$, where $\chi_{A_{k}}$ is the characteristic function of $A_{k}$. 
Definition 1.4. ([5]) Let $\alpha \in \mathbb{R}, 0<p \leq \infty$ and $q(\cdot) \in \mathcal{P}\left(\mathbb{R}^{n}\right)$. The homogeneous Herz space $\dot{K}_{q(\cdot)}^{\alpha, p}\left(\mathbb{R}^{n}\right)$ is defined by

$$
\dot{K}_{q(\cdot)}^{\alpha, p}\left(\mathbb{R}^{n}\right)=\left\{f \in L_{\text {loc }}^{q(\cdot)}\left(\mathbb{R}^{n} \backslash\{0\}\right):\|f\|_{\dot{K}_{q(\cdot)}^{\alpha, p}\left(\mathbb{R}^{n}\right)}<\infty\right\},
$$

where

$$
\|f\|_{\dot{K}_{q(\cdot)}^{\alpha, p}\left(\mathbb{R}^{n}\right)}=\left\{\sum_{k \in \mathbb{Z}} 2^{k \alpha p}\left\|f \chi_{k}\right\|_{L^{q(\cdot)}\left(\mathbb{R}^{n}\right)}^{p}\right\}^{1 / p} .
$$

The non-homogeneous Herz space $K_{q(\cdot)}^{\alpha, p}\left(\mathbb{R}^{n}\right)$ is defined by

$$
K_{q(\cdot)}^{\alpha, p}\left(\mathbb{R}^{n}\right)=\left\{f \in L_{\mathrm{loc}}^{q(\cdot)}\left(\mathbb{R}^{n}\right):\|f\|_{K_{q(\cdot)}^{\alpha, p}\left(\mathbb{R}^{n}\right)}<\infty\right\}
$$

where

$$
\|f\|_{K_{q(\cdot)}^{\alpha, p}\left(\mathbb{R}^{n}\right)}=\left\{\sum_{k=0}^{\infty} 2^{k \alpha p}\left\|f \tilde{\chi}_{k}\right\|_{L^{q(\cdot)}\left(\mathbb{R}^{n}\right)}^{p}\right\}^{1 / p} .
$$

In [10], we gave the definition of Herz-type Hardy space with variable exponent $H \dot{K}_{q(\cdot)}^{\alpha, p}\left(\mathbb{R}^{n}\right) . \mathcal{S}\left(\mathbb{R}^{n}\right)$ denotes the space of Schwartz functions, and $\mathcal{S}^{\prime}\left(\mathbb{R}^{n}\right)$ denotes the dual space of $\mathcal{S}\left(\mathbb{R}^{n}\right)$. Let $G_{N} f(x)$ be the grand maximal function of $f(x)$ defined by

$$
G_{N} f(x)=\sup _{\phi \in \mathcal{A}_{N}}\left|\phi_{\nabla}^{*}(f)(x)\right|,
$$

where $\mathcal{A}_{N}=\left\{\phi \in \mathcal{S}\left(\mathbb{R}^{n}\right): \sup _{|\alpha|,|\beta| \leq N}\left|x^{\alpha} D^{\beta} \phi(x)\right| \leq 1\right\}$ and $N>n+1, \phi_{\nabla}^{*}$ is the nontangential maximal operator defined by

$$
\phi_{\nabla}^{*}(f)(x)=\sup _{|y-x|<t}\left|\phi_{t} * f(y)\right|
$$

with $\phi_{t}(x)=t^{-n} \phi(x / t)$.

Definition 1.5. ([10]) Let $\alpha \in \mathbb{R}, 0<p<\infty, q(\cdot) \in \mathcal{P}\left(\mathbb{R}^{n}\right)$ and $N>n+1$.

(i) The homogeneous Herz-type Hardy space $H \dot{K}_{q(\cdot)}^{\alpha, p}\left(\mathbb{R}^{n}\right)$ is defined by

$$
H \dot{K}_{q(\cdot)}^{\alpha, p}\left(\mathbb{R}^{n}\right)=\left\{f \in \mathcal{S}^{\prime}\left(\mathbb{R}^{n}\right): G_{N} f(x) \in \dot{K}_{q(\cdot)}^{\alpha, p}\left(\mathbb{R}^{n}\right)\right\}
$$

and we define $\|f\|_{H \dot{K}_{q(\cdot)}^{\alpha, p}\left(\mathbb{R}^{n}\right)}=\left\|G_{N} f\right\|_{\dot{K}_{q(\cdot)}^{\alpha, p}\left(\mathbb{R}^{n}\right)}$.

(ii) The non-homogeneous Herz-type Hardy space $H K_{q(\cdot)}^{\alpha, p}\left(\mathbb{R}^{n}\right)$ is defined by

$$
H K_{q(\cdot)}^{\alpha, p}\left(\mathbb{R}^{n}\right)=\left\{f \in \mathcal{S}^{\prime}\left(\mathbb{R}^{n}\right): G_{N} f(x) \in K_{q(\cdot)}^{\alpha, p}\left(\mathbb{R}^{n}\right)\right\}
$$

and we define $\|f\|_{H K_{q(\cdot)}^{\alpha, p}\left(\mathbb{R}^{n}\right)}=\left\|G_{N} f\right\|_{K_{q(\cdot)}^{\alpha, p}\left(\mathbb{R}^{n}\right)}$.

For $\alpha \in \mathbb{R}$ we denote by $[\alpha]$ the largest integer less than or equal to $\alpha$.

Definition 1.6. ([10]) Let $n \delta_{2} \leq \alpha<\infty, q(\cdot) \in \mathcal{P}\left(\mathbb{R}^{n}\right)$, and $s \geq\left[\alpha-n \delta_{2}\right]$.

(i) A function $a(x)$ on $\mathbb{R}^{n}$ is said to be a central $(\alpha, q(\cdot))$-atom, if it satisfies

(1) supp $a \subset B(0, r)=\left\{x \in \mathbb{R}^{n}:|x|<r\right\}$.

(2) $\|a\|_{L^{q(\cdot)}\left(\mathbb{R}^{n}\right)} \leq|B(0, r)|^{-\alpha / n}$.

(3) $\int_{\mathbb{R}^{n}} a(x) x^{\beta} d x=0,|\beta| \leq s$. 
(ii) A function $a(x)$ on $\mathbb{R}^{n}$ is said to be a central $(\alpha, q(\cdot))$-atom of restricted type, if it satisfies the conditions (2), (3) above and

$(1)^{\prime} \operatorname{supp} a \subset B(0, r), r \geq 1$.

Lemma 1.7. ([10]) Let $n \delta_{2} \leq \alpha<\infty, 0<p<\infty$ and $q(\cdot) \in \mathcal{B}\left(\mathbb{R}^{n}\right)$. Then $f \in H \dot{K}_{q(\cdot)}^{\alpha, p}\left(\mathbb{R}^{n}\right)\left(\right.$ or $\left.H K_{q(\cdot)}^{\alpha, p}\left(\mathbb{R}^{n}\right)\right)$ if and only if

$$
f=\sum_{k=-\infty}^{\infty} \lambda_{k} a_{k}\left(\text { or } \sum_{k=0}^{\infty} \lambda_{k} a_{k}\right), \quad \text { in the sense of } \mathcal{S}^{\prime}\left(\mathbb{R}^{n}\right)
$$

where each $a_{k}$ is a central $(\alpha, q(\cdot))$-atom (or central $(\alpha, q(\cdot))$-atom of restricted type) with support contained in $B_{k}$ and $\sum_{k=-\infty}^{\infty}\left|\lambda_{k}\right|^{p}<\infty\left(\right.$ or $\left.\sum_{k=0}^{\infty}\left|\lambda_{k}\right|^{p}<\infty\right)$. Moreover,

$$
\|f\|_{H \dot{K}_{q(\cdot)}^{\alpha, p}\left(\mathbb{R}^{n}\right)} \approx \inf \left(\sum_{k=-\infty}^{\infty}\left|\lambda_{k}\right|^{p}\right)^{1 / p}\left(\text { or }\|f\|_{H K_{q(\cdot)}^{\alpha, p}\left(\mathbb{R}^{n}\right)} \approx \inf \left(\sum_{k=0}^{\infty}\left|\lambda_{k}\right|^{p}\right)^{1 / p}\right),
$$

where the infimum is taken over all above decomposition of $f$.

In [11], we gave some real-variable characterizations for $H \dot{K}_{q(\cdot)}^{\alpha, p}\left(\mathbb{R}^{n}\right)$ and $H K_{q(\cdot)}^{\alpha, p}\left(\mathbb{R}^{n}\right)$. Let $\phi \in \mathcal{S}\left(\mathbb{R}^{n}\right)$ with integral 1. For $t>0$, set $\phi_{t}(x)=t^{-n} \phi(x / t)$. For $f \in \mathcal{S}^{\prime}\left(\mathbb{R}^{n}\right)$, define the maximal operator $\phi_{+}^{*}, \phi_{\nabla, N}^{*}\left(\right.$ with $N>1$ ) and $\phi_{M}^{* *}($ with $M \in \mathbb{N}$ ) by

$$
\phi_{+}^{*}(f)(x)=\sup _{t>0}\left|\left(f * \phi_{t}\right)(x)\right|, \phi_{\nabla, N}^{*}(f)(x)=\sup _{t>0} \sup _{|x-y|<N t}\left|\left(f * \phi_{t}\right)(y)\right|
$$

and

$$
\phi_{M}^{* *}(f)(x)=\sup _{(y, t) \in \mathbb{R}_{+}^{n+1}}\left|\left(f * \phi_{t}\right)(y)\right|\left(\frac{t}{|x-y|+t}\right)^{M}
$$

Lemma 1.8. ([11]) Let $0<\alpha<\infty, 0<p<\infty$ and $q(\cdot) \in \mathcal{B}\left(\mathbb{R}^{n}\right)$. For $f \in \mathcal{S}^{\prime}\left(\mathbb{R}^{n}\right)$, the following statements are equivalent:

(i) $f \in H \dot{K}_{q(\cdot)}^{\alpha, p}\left(\mathbb{R}^{n}\right) \quad\left(\right.$ or $\left.H K_{q(\cdot)}^{\alpha, p}\left(\mathbb{R}^{n}\right)\right)$.

(ii) For some $N>1, \phi_{\nabla, N}^{*}(f) \in \dot{K}_{q(\cdot)}^{\alpha, p}\left(\mathbb{R}^{n}\right) \quad\left(\right.$ or $\left.K_{q(\cdot)}^{\alpha, p}\left(\mathbb{R}^{n}\right)\right)$.

(iii) $\phi_{\nabla}^{*}(f) \in \dot{K}_{q(\cdot)}^{\alpha, p}\left(\mathbb{R}^{n}\right)$ (or $\left.K_{q(\cdot)}^{\alpha, p}\left(\mathbb{R}^{n}\right)\right)$.

(iv) $\phi_{+}^{*}(f) \in \dot{K}_{q(\cdot)}^{\alpha, p}\left(\mathbb{R}^{n}\right) \quad\left(\right.$ or $\left.K_{q(\cdot)}^{\alpha, p}\left(\mathbb{R}^{n}\right)\right)$.

Moreover,

$$
\|f\|_{H \dot{K}_{q(\cdot)}^{\alpha, p}\left(\mathbb{R}^{n}\right)} \approx\left\|\phi_{\nabla, N}^{*}(f)\right\|_{\dot{K}_{q(\cdot)}^{\alpha, p}\left(\mathbb{R}^{n}\right)} \approx\left\|\phi_{\nabla}^{*}(f)\right\|_{\dot{K}_{q(\cdot)}^{\alpha, p}\left(\mathbb{R}^{n}\right)} \approx\left\|\phi_{+}^{*}(f)\right\|_{\dot{K}_{q(\cdot)}^{\alpha, p}\left(\mathbb{R}^{n}\right)}
$$

and

$$
\|f\|_{H K_{q(\cdot)}^{\alpha, p}\left(\mathbb{R}^{n}\right)} \approx\left\|\phi_{\nabla, N}^{*}(f)\right\|_{K_{q(\cdot)}^{\alpha, p}\left(\mathbb{R}^{n}\right)} \approx\left\|\phi_{\nabla}^{*}(f)\right\|_{K_{q(\cdot)}^{\alpha, p}\left(\mathbb{R}^{n}\right)} \approx\left\|\phi_{+}^{*}(f)\right\|_{K_{q(\cdot)}^{\alpha, p}\left(\mathbb{R}^{n}\right)} .
$$

In [9], we establish the block decomposition for the Herz spaces with variable exponent. 
Definition 1.9. ([9]) Let $0<\alpha<\infty, q(\cdot) \in \mathcal{P}\left(\mathbb{R}^{n}\right)$.

(i) A function $a(x)$ on $\mathbb{R}^{n}$ is said to be a central $(\alpha, q(\cdot))$-block if

(1) $\operatorname{supp} a \subset B(0, r)=\left\{x \in \mathbb{R}^{n}:|x|<r\right\}$.

(2) $\|a\|_{L^{q(\cdot)\left(\mathbb{R}^{n}\right)}} \leq C r^{-\alpha}$.

(ii) A function $a(x)$ on $\mathbb{R}^{n}$ is said to be a central $(\alpha, q(\cdot))$-block of restricted type if

(1) $\operatorname{supp} a \subset B(0, r)$ for some $r \geq 1$.

(2) $\|a\|_{L^{q(\cdot)\left(\mathbb{R}^{n}\right)}} \leq r^{-\alpha}$.

Inspired by $[3,10]$, we introduce a certain Herz-type Hardy space with variable exponent and establish the atom decomposition theorem for it. Using this decomposition, we obtain some boundedness on the Herz-type Hardy space with variable exponent for a class of pseudo-differential operators.

\section{MAIN RESUlTS AND THEIR PROOFS}

In this section, we will give the definition of local Herz-type Hardy spaces with variable exponent $h \dot{K}_{q(\cdot)}^{\alpha, p}\left(\mathbb{R}^{n}\right)$ and $h K_{q(\cdot)}^{\alpha, p}\left(\mathbb{R}^{n}\right)$ firstly.

Definition 2.1. Let $n \delta_{2} \leq \alpha<\infty, 0<p \leq \infty, q(\cdot) \in \mathcal{B}\left(\mathbb{R}^{n}\right)$.

(i)A function $f \in L_{\text {loc }}^{q(\cdot)}\left(\mathbb{R}^{n} \backslash\{0\}\right)$ is said to be in the space $h \dot{K}_{q(\cdot)}^{\alpha, p}\left(\mathbb{R}^{n}\right)$ if for some $N \in \mathbb{N}$ with $N>\alpha / \delta_{2}+1$, the maximal function

$$
\tilde{G}_{N}(f)=\sup _{\phi \in \mathcal{A}_{N}} \sup _{0<t<1,|x-y|<t}\left|f * \phi_{t}(y)\right|
$$

belongs to the space $\dot{K}_{q(\cdot)}^{\alpha, p}\left(\mathbb{R}^{n}\right) ;$ where $\mathcal{A}_{N}=\left\{\phi \in \mathcal{S}\left(\mathbb{R}^{n}\right): \sup _{|\alpha|,|\beta| \leq N}\left|x^{\alpha} D^{\beta} \phi(x)\right| \leq\right.$ $1\}$ and $\phi_{t}(x)=t^{-n} \phi(x / t)$.

(ii)A function $f$ is said to be in the space $h K_{q(\cdot)}^{\alpha, p}\left(\mathbb{R}^{n}\right)$ if $\tilde{G}_{N}(f) \in K_{q(\cdot)}^{\alpha, p}\left(\mathbb{R}^{n}\right)$.

Moreover, we define that $\|f\|_{h \dot{K}_{q(\cdot)}^{\alpha, p}\left(\mathbb{R}^{n}\right)}=\left\|\tilde{G}_{N}(f)\right\|_{\dot{K}_{q(\cdot)}^{\alpha, p}\left(\mathbb{R}^{n}\right)}$ and $\|f\|_{h K_{q(\cdot)}^{\alpha, p}\left(\mathbb{R}^{n}\right)}=$ $\left\|\tilde{G}_{N}(f)\right\|_{K_{q(\cdot)}^{\alpha, p}\left(\mathbb{R}^{n}\right)}$.

It should be pointed out that, the spaces $h \dot{K}_{q(\cdot)}^{\alpha, p}\left(\mathbb{R}^{n}\right)$ and $h K_{q(\cdot)}^{\alpha, p}\left(\mathbb{R}^{n}\right)$ also enjoy the characterizations in terms of the maximal operator, which are similar to those of the spaces $H \dot{K}_{q(\cdot)}^{\alpha, p}\left(\mathbb{R}^{n}\right)$ and $H K_{q(\cdot)}^{\alpha, p}\left(\mathbb{R}^{n}\right)$, namely,

Theorem 2.2. Let $n \delta_{2} \leq \alpha<\infty, 0<p<\infty, q(\cdot) \in \mathcal{B}\left(\mathbb{R}^{n}\right), \phi \in \mathcal{S}\left(\mathbb{R}^{n}\right)$ with $\int_{\mathbb{R}^{n}} \phi(x) d x=1$. For $f \in \mathcal{S}^{\prime}\left(\mathbb{R}^{n}\right)$, the following statements are equivalent:

(i) $f \in h \dot{K}_{q(\cdot)}^{\alpha, p}\left(\mathbb{R}^{n}\right) \quad\left(\right.$ or $\left.h K_{q(\cdot)}^{\alpha, p}\left(\mathbb{R}^{n}\right)\right)$.

(ii) $\tilde{\phi}_{\nabla}^{*}(f)=\sup _{0<t<1} \sup _{|x-y|<t}\left|f * \phi_{t}(y)\right| \in \dot{K}_{q(\cdot)}^{\alpha, p}\left(\mathbb{R}^{n}\right) \quad\left(\right.$ or $\left.K_{q(\cdot)}^{\alpha, p}\left(\mathbb{R}^{n}\right)\right)$.

(iii) $\tilde{\phi}_{+}^{*}(f)=\sup _{0<t<1}\left|f * \phi_{t}(x)\right| \in \dot{K}_{q(\cdot)}^{\alpha, p}\left(\mathbb{R}^{n}\right) \quad\left(\right.$ or $\left.K_{q(\cdot)}^{\alpha, p}\left(\mathbb{R}^{n}\right)\right)$.

Moreover,

$$
\|f\|_{h \dot{K}_{q(\cdot)}^{\alpha, p}\left(\mathbb{R}^{n}\right)} \approx\left\|\tilde{\phi}_{\nabla}^{*}(f)\right\|_{\dot{K}_{q(\cdot)}^{\alpha, p}\left(\mathbb{R}^{n}\right)} \approx\left\|\tilde{\phi}_{+}^{*}(f)\right\|_{\dot{K}_{q(\cdot)}^{\alpha, p}\left(\mathbb{R}^{n}\right)}
$$

and

$$
\|f\|_{h K_{q(\cdot)}^{\alpha, p}\left(\mathbb{R}^{n}\right)} \approx\left\|\tilde{\phi}_{\nabla}^{*}(f)\right\|_{K_{q(\cdot)}^{\alpha, p}\left(\mathbb{R}^{n}\right)} \approx\left\|\tilde{\phi}_{+}^{*}(f)\right\|_{K_{q(\cdot)}^{\alpha, p}\left(\mathbb{R}^{n}\right)}
$$


Proof. The method of proof is similar to Lemma 1.8. Here we omit it.

To give another characterization of $h \dot{K}_{q(\cdot)}^{\alpha, p}\left(\mathbb{R}^{n}\right)$, we first give a preliminary lemma.

Theorem 2.3. Let $n \delta_{2} \leq \alpha<\infty, 0<p<\infty$ and $q(\cdot) \in \mathcal{B}\left(\mathbb{R}^{n}\right)$. Suppose that $\phi \in \mathcal{S}\left(\mathbb{R}^{n}\right)$ such that

$$
\int_{\mathbb{R}^{n}} \phi(x) d x=1, \int_{\mathbb{R}^{n}} x^{\beta} \phi(x) d x=0, \text { for all } \beta \in \mathbb{N}^{n},|\beta| \leq N .
$$

Then

$$
\|f-\phi * f\|_{H \dot{K}_{q(\cdot)}^{\alpha, p}\left(\mathbb{R}^{n}\right)} \leq C\|f\|_{h \dot{K}_{q(\cdot)}^{\alpha, p}\left(\mathbb{R}^{n}\right)} .
$$

Moreover, if $f \in h \dot{K}_{q(\cdot)}^{\alpha, p}\left(\mathbb{R}^{n}\right)$, then $f-\phi * f \in H \dot{K}_{q(\cdot)}^{\alpha, p}\left(\mathbb{R}^{n}\right)$.

Proof. Take $\psi \in \mathcal{S}\left(\mathbb{R}^{n}\right)$ with $\int_{\mathbb{R}^{n}} \psi(x) d x=1$. By Lemma 1.8, we see that

$$
\begin{aligned}
\|f-\phi * f\|_{H \dot{K}_{q(\cdot)}^{\alpha, p}\left(\mathbb{R}^{n}\right)}= & C\left\|\sup _{t>0}\left|\psi_{t} *(f-\phi * f)\right|\right\|_{\dot{K}_{q, \cdot)}^{\alpha, p}\left(\mathbb{R}^{n}\right)} \\
\leq & C\left\|\sup _{1>t>0}\left|\psi_{t} * f\right|\right\|_{\dot{K}_{q(\cdot)}^{\alpha, p}\left(\mathbb{R}^{n}\right)}+C\left\|\sup _{1>t>0}\left|\psi_{t} * \phi * f\right|\right\|_{\dot{K}_{q(\cdot)}^{\alpha, p}\left(\mathbb{R}^{n}\right)} \\
& +C\left\|\sup _{\infty>t \geq 1}\left|\psi_{t} *(f-\phi * f)\right|\right\|_{\dot{K}_{q(\cdot)}^{\alpha, p}\left(\mathbb{R}^{n}\right)} \\
= & U_{1}+U_{2}+U_{3} .
\end{aligned}
$$

Note that $\sup _{0<t<1}\left|\psi_{t} * f\right| \leq C \tilde{G}_{N}(f)(x)$. So by Definition 2.1 we have

$$
U_{1} \leq C\left\|\tilde{G}_{N}(f)\right\|_{\dot{K}_{q(\cdot)}^{\alpha, p}\left(\mathbb{R}^{n}\right)}=C\|f\|_{h \dot{K}_{q(\cdot)}^{\alpha, p}\left(\mathbb{R}^{n}\right)} \cdot
$$

To estimate $U_{2}$, we first claim that there is a constant $C_{0}$ independent of $t \leq 1$ such that $C_{0}^{-1} \psi_{t} * \phi \in \mathcal{A}_{N}$. In fact, for any $\gamma$ with $|\gamma| \leq n+N$ and any $\beta$ with $|\beta| \leq N$, a trivial computation leads to that

$$
\begin{aligned}
\left|x^{\gamma} D^{\beta}\left(\psi_{t} * \phi\right)(x)\right| \leq & |x|^{|\gamma|}\left|\int_{2|y|<|x|} \frac{1}{t^{n}} \psi\left(\frac{y}{t}\right) D^{\beta} \phi(x-y) d y\right| \\
& +|x|^{|\gamma|}\left|\int_{2|y| \geq|x|} \frac{1}{t^{n}} \psi\left(\frac{y}{t}\right) D^{\beta} \phi(x-y) d y\right| \\
\leq & C|x|^{|\gamma|} \int_{2|y|<|x|}\left|\psi\left(t^{-1} y\right)\right| \frac{d y}{(1+|x-y|)^{|\gamma|}} \\
& +C|x|^{|\gamma|} t^{-n} \int_{2|y| \geq|x|} \frac{t^{|\gamma|}}{|y|^{|\gamma|}} d y \\
\leq & C\|\psi\|_{L^{1}\left(\mathbb{R}^{n}\right)}+\left.C|| y\right|^{|\gamma|} \psi \|_{L^{1}\left(\mathbb{R}^{n}\right)} \leq C_{0},
\end{aligned}
$$

which means that $C_{0}^{-1}\left(\psi_{t} * \phi\right) \in \mathcal{A}_{N}\left(\mathbb{R}^{n}\right)$. Thus,

$$
\left|\psi_{t} * \phi * f(x)\right| \leq C \tilde{G}_{N}(f)(x)
$$

and

$$
U_{2} \leq C\|f\|_{h \dot{K}_{q(\cdot)}^{\alpha, p}\left(\mathbb{R}^{n}\right)}
$$


On the other hand, a cumbersome but straightforward computation shows that for any fixed $k \geq 1$, any multi-index $\beta$ and any $t \geq 1$,

$$
\left|D^{\beta}\left(\psi_{t}-\phi * \psi_{t}\right)\right| \leq C_{k, \beta}(1+|x|)^{-k},
$$

which via a trivial computation leads to that

$$
\sup _{1 \leq t<\infty}\left|\psi_{t} *(f-\phi * f)(x)\right| \leq C \tilde{G}_{N}(f)(x) .
$$

Thus we get that

$$
U_{3} \leq C\left\|\tilde{G}_{N}(f)\right\|_{\dot{K}_{q(\cdot)}^{\alpha, p}\left(\mathbb{R}^{n}\right)}=C\|f\|_{h \dot{K}_{q(\cdot)}^{\alpha, p}\left(\mathbb{R}^{n}\right)} .
$$

This completes the proof of Theorem 2.3.

Next, we will give the atomic decomposition for $h \dot{K}_{q(\cdot)}^{\alpha, p}\left(\mathbb{R}^{n}\right)$.

Theorem 2.4. Let $n \delta_{2} \leq \alpha<\infty, 0<p<\infty$ and $q(\cdot) \in \mathcal{B}\left(\mathbb{R}^{n}\right)$. Then $f \in$ $h \dot{K}_{q(\cdot)}^{\alpha, p}\left(\mathbb{R}^{n}\right)$ if and only if

$$
f(x)=\sum_{k=-\infty}^{\infty} \lambda_{k} a_{k}(x), \quad \text { in the sense of } \mathcal{S}^{\prime}\left(\mathbb{R}^{n}\right),
$$

where for $k \leq 0, a_{k}$ is a central $(\alpha, q(\cdot))$-atom, while for $k>0, a_{k}$ is a central $(\alpha, q(\cdot))$-block, and $\left\{\lambda_{k}\right\}_{k=-\infty}^{\infty}$ satisfies $\sum_{k=-\infty}^{\infty}\left|\lambda_{k}\right|^{p}<\infty$. Moreover,

$$
\|f\|_{h \dot{K}_{q(\cdot)}^{\alpha, p}\left(\mathbb{R}^{n}\right)} \approx \inf \left(\sum_{k=-\infty}^{\infty}\left|\lambda_{k}\right|^{p}\right)^{1 / p}
$$

where the infimum is taken over all above decomposition of $f$.

Proof. To prove the necessity, let $f \in h \dot{K}_{q(\cdot)}^{\alpha, p}\left(\mathbb{R}^{n}\right)$ and $\phi \in \mathcal{S}\left(\mathbb{R}^{n}\right)$ be the same as in Theorem 2.3. Then $g=f-\phi * f \in H \dot{K}_{q(\cdot)}^{\alpha, p}\left(\mathbb{R}^{n}\right)$, which implies that

$$
f=g+\phi * f, \quad \text { in the sense of } \mathcal{S}^{\prime}\left(\mathbb{R}^{n}\right) .
$$

For $g$, we have the central $(\alpha, q(\cdot))$-atom decomposition by Lemma 1.7. Thus, it suffices to decompose $\phi * f$.

Let $\psi$ be a radial smooth function such that $\operatorname{supp} \psi \subset\{x: 1 / 2-\varepsilon \leq|x| \leq 1+\varepsilon\}$ with $0<\varepsilon<1 / 4$, and $\psi(x)=1$ if $1 / 2 \leq|x| \leq 1$. Set $\psi_{k}(x)=\phi\left(2^{-k} x\right)$ for $k \in \mathbb{Z}$ and $\tilde{A}_{k, \varepsilon}=\left\{x: 2^{k-1}-2^{k} \varepsilon \leq|x| \leq 2^{k}+2^{k} \varepsilon\right\}$. We know that

$$
\operatorname{supp} \psi \subset \tilde{A}_{k, \varepsilon}, \quad \psi_{k}(x)=1 \text { if } x \in A_{k}=\left\{x: 2^{k-1}<|x| \leq 2^{k}\right\} .
$$

Obviously, $1 \leq \sum_{k=-\infty}^{\infty} \psi_{k}(x) \leq 2,|x|>0$. Let

$$
\Phi_{k}(x)=\left\{\begin{array}{l}
\psi_{k}(x) / \sum_{l=-\infty}^{\infty} \psi_{l}(x), \quad x \neq 0 \\
0, \quad x=0
\end{array}\right.
$$


then $\sum_{k=-\infty}^{\infty} \Phi_{k}(x)=1$ for $x \neq 0$. For some $m \in \mathbb{Z}_{+}$, we denote by $\mathcal{P}_{m}$ the class of all the real polynomials with the degree less than $m$. Let $P_{k}(x)=P_{\tilde{A}_{k, \varepsilon}}((f *$ $\left.\phi) \Phi_{k}\right)(x) \chi_{\tilde{A}_{k, \varepsilon}}(x) \in \mathcal{P}_{m}\left(\mathbb{R}^{n}\right)$ be the unique polynomial such that

$$
\int_{\tilde{A}_{k, \varepsilon}}\left((f * \phi)(x) \Phi_{k}(x)-P_{k}(x)\right) x^{\beta} d x=0, \quad|\beta| \leq m=\left[\alpha-n \delta_{2}\right] .
$$

Write

$f * \phi(x)=\sum_{k=-\infty}^{\infty}\left(f * \phi(x) \Phi_{k}(x)-P_{k}(x)\right)+\sum_{k=-\infty}^{\infty} P_{k}(x)=\sum_{k=-\infty}^{\infty} H_{k}^{1}(x)+\sum_{k=-\infty}^{\infty} H_{k}^{2}(x)$.

For the term $\sum_{k=-\infty}^{\infty} H_{k}^{1}(x)$, let $\left\{\phi_{l}^{k}:|l| \leq m\right\}$ be the orthogonal polynomials limited on $\tilde{A}_{k, \varepsilon}$ with respect to the weight $\left|\tilde{A}_{k, \varepsilon}\right|^{-1}$, which are obtained from $\left\{x^{\beta}:|\beta| \leq m\right\}$ by the Gram-Schmidt method, that is

$$
\left\langle\phi_{\nu}^{k}, \phi_{\mu}^{k}\right\rangle=\frac{1}{\left|\tilde{A}_{k, \varepsilon}\right|} \int_{\tilde{A}_{k, \varepsilon}} \phi_{\nu}^{k}(x) \phi_{\mu}^{k}(x) d x=\delta_{\nu \mu}
$$

It is easy to see that

$$
P_{k}(x)=\sum_{|l| \leq m}\left\langle(f * \phi) \Phi_{k}, \phi_{l}^{k}\right\rangle \phi_{l}^{k}(x), \quad x \in \tilde{A}_{k, \varepsilon} .
$$

On the other hand, from

$$
\frac{1}{\left|\tilde{A}_{k, \varepsilon}\right|} \int_{\tilde{A}_{k, \varepsilon}} \phi_{\nu}^{k}(x) \phi_{\mu}^{k}(x) d x=\delta_{\nu \mu}
$$

we infer that

$$
\frac{1}{\left|\tilde{A}_{1, \varepsilon}\right|} \int_{\tilde{A}_{1, \varepsilon}} \phi_{\nu}^{k}\left(2^{k-1} y\right) \phi_{\mu}^{k}\left(2^{k-1} y\right) d y=\delta_{\nu \mu} .
$$

It then follows directly that $\phi_{\nu}^{k}\left(2^{k-1} y\right)=\phi_{\nu}^{1}(y), y \in \tilde{A}_{1, \varepsilon}$. That is $\phi_{\nu}^{k}(x)=$ $\phi_{\nu}^{1}\left(2^{-(k-1)} x\right)$ for $x \in \tilde{A}_{k, \varepsilon}$. Thus $\left|\phi_{\nu}^{k}(x)\right| \leq C$, and for $x \in \tilde{A}_{k, \varepsilon}$, by the generalized Hölder inequality we have

$$
\begin{aligned}
\left|P_{k}(x)\right| & \leq \frac{C}{\left|\tilde{A}_{k, \varepsilon}\right|} \int_{\tilde{A}_{k, \varepsilon}}\left|(f * \phi)(x) \Phi_{k}(x)\right| d x \\
& \leq \frac{C}{\left|\tilde{A}_{k, \varepsilon}\right|}\left\|(f * \phi) \Phi_{k}\right\|_{L^{q(\cdot)}\left(\mathbb{R}^{n}\right)}\left\|\chi_{\tilde{A}_{k, \varepsilon}}\right\|_{L^{q^{\prime}(\cdot)}\left(\mathbb{R}^{n}\right)}
\end{aligned}
$$


Therefore, by Lemma 1.3 we have that

$$
\begin{aligned}
\left\|H_{k}^{1}\right\|_{L^{q(\cdot)}\left(\mathbb{R}^{n}\right)} \leq & \left\|(f * \phi) \Phi_{k}\right\|_{L^{q(\cdot)}\left(\mathbb{R}^{n}\right)}+\left\|P_{k}\right\|_{L^{q(\cdot)}\left(\mathbb{R}^{n}\right)} \\
\leq & \left\|(f * \phi) \Phi_{k}\right\|_{L^{q(\cdot)}\left(\mathbb{R}^{n}\right)} \\
& +\frac{C}{\left|\tilde{A}_{k, \varepsilon}\right|}\left\|(f * \phi) \Phi_{k}\right\|_{L^{q(\cdot)}\left(\mathbb{R}^{n}\right)}\left\|\chi_{\tilde{A}_{k, \varepsilon}}\right\|_{L^{q^{(\cdot \cdot)}\left(\mathbb{R}^{n}\right)}}\left\|\chi_{\tilde{A}_{k, \varepsilon}}\right\|_{L^{q(\cdot)}\left(\mathbb{R}^{n}\right)} \\
\leq & \left\|(f * \phi) \Phi_{k}\right\|_{L^{q(\cdot)\left(\mathbb{R}^{n}\right)}}+C\left\|(f * \phi) \Phi_{k}\right\|_{L^{q(\cdot)}\left(\mathbb{R}^{n}\right)} \\
\leq & C(f * \phi) \Phi_{k} \|_{L^{q(\cdot)\left(\mathbb{R}^{n}\right)}} \\
\leq & C \sum_{j=k-1}^{k+1}\left\|\tilde{G}_{N}(f) \chi_{j}\right\|_{L^{q(\cdot)}\left(\mathbb{R}^{n}\right)} .
\end{aligned}
$$

Set $\lambda_{k}^{1}=2^{(k+1) \alpha}\left\|H_{k}^{1}\right\|_{L^{q(\cdot)}\left(\mathbb{R}^{n}\right)}$ and $b_{k}(x)=2^{-(k+1) \alpha}\left\|H_{k}^{1}\right\|_{L^{q(\cdot)}\left(\mathbb{R}^{n}\right)}^{-1} H_{k}^{1}(x)$. It is easy to verify that each $b_{k}$ is central $(\alpha, q(\cdot))$-atom and $\sum_{k=-\infty}^{\infty} H_{k}^{1}(x)=\sum_{k=-\infty}^{\infty} \lambda_{k} a_{k}(x)$. Moreover,

$$
\begin{aligned}
\sum_{k=-\infty}^{\infty}\left|\lambda_{k}\right|^{p} & =\sum_{k=-\infty}^{\infty} 2^{(k+1) \alpha p}\left\|H_{k}^{1}\right\|_{L^{q(\cdot)}\left(\mathbb{R}^{n}\right)}^{p} \\
& \leq C \sum_{k=-\infty}^{\infty} 2^{k \alpha p}\left\|\tilde{G}_{N}(x) \chi_{k}\right\|_{L^{q(\cdot)}\left(\mathbb{R}^{n}\right)} \\
& =C\|f\|_{h \dot{K}_{q(\cdot)}^{\alpha, p}\left(\mathbb{R}^{n}\right)}^{p}
\end{aligned}
$$

We now turn our attention to $\sum_{k=-\infty}^{\infty} H_{k}^{2}(x)$. Let $\left\{\psi_{l}^{k}:|l| \leq m\right\}$ be the dual basis of $\left\{x^{\beta}:|\beta| \leq m\right\}$ limited on $\tilde{A}_{k}$ with respected to the weight $\left|\tilde{A}_{k, \varepsilon}\right|^{-1}$, that is,

$$
\left\langle\psi_{l}^{k}, x^{\beta}\right\rangle=\frac{1}{\tilde{A}_{k, \varepsilon}} \int_{\tilde{A}_{k, \varepsilon}} x^{\beta} \psi_{l}^{k}(x) d x=\delta_{\beta l} .
$$

We can prove that if $\phi_{l}^{k}(x)=\sum_{|\nu| \leq m} \beta_{l \nu}^{k} x^{\nu}$, then $\psi_{l}^{k}(x)=\sum_{|\nu| \leq m} \beta_{\nu l}^{k} \phi_{\nu}^{k}(x)$. In fact, let $\psi_{l}^{k}(x)=\sum_{|\nu| \leq m} C_{\nu l}^{k} \phi_{\nu}^{k}(x)$, then

$$
C_{\nu l}^{k}=\left\langle\psi_{l}^{k}, \phi_{\nu}^{k}\right\rangle=\left\langle\psi_{l}^{k}, \sum_{|\gamma| \leq m} \beta_{\nu \gamma}^{k} x^{\gamma}\right\rangle=\sum_{|\gamma| \leq m} \beta_{\nu \gamma}^{k}\left\langle\psi_{l}^{k}, x^{\gamma}\right\rangle=\beta_{\nu l}^{k}
$$


Thus, for $x \in \tilde{A}_{k, \varepsilon}$,

$$
\begin{aligned}
P_{k}(x) & =\sum_{|\nu| \leq m}\left\langle(f * \phi) \Phi_{k}, \phi_{\nu}^{k}\right\rangle \phi_{\nu}^{k}(x) \\
& =\sum_{|\nu| \leq m}^{\mid l}\left\langle(f * \phi) \Phi_{k}, \sum_{|l| \leq m} \beta_{\nu l}^{k} y^{l}\right\rangle \phi_{\nu}^{k}(x) \\
& =\sum_{|l| \leq m}\left\langle(f * \phi) \Phi_{k}, y^{l}\right\rangle \sum_{|\nu| \leq m} \beta_{\nu l}^{k} \phi_{\nu}^{k}(x) \\
& =\sum_{|l| \leq m}\left\langle(f * \phi) \Phi_{k}, y^{l}\right\rangle \psi_{l}^{k}(x) .
\end{aligned}
$$

It is easy to prove that if $x \in \tilde{A}_{k, \varepsilon}$, then $\left|\psi_{l}^{k}(x)\right| \leq C 2^{-k|l|}$ (see [7, Theorem 2.1]). It follows that

$$
\begin{aligned}
\sum_{k=-\infty}^{\infty} H_{k}^{2}(x)= & \sum_{k=-\infty}^{\infty} P_{k}(x) \\
= & \sum_{k=-\infty}^{\infty} \sum_{|l| \leq m}\left\langle(f * \phi) \Phi_{k}, y^{l}\right\rangle \psi_{l}^{k}(x) \chi_{\tilde{A}_{k, \varepsilon}}(x) \\
= & \sum_{|l| \leq m} \sum_{k=-\infty}^{\infty}\left(\int_{\mathbb{R}^{n}}(f * \phi)(y) \Phi_{k}(y) y^{l} d y\right) \frac{\psi_{l}^{k}(x) \chi_{\tilde{A}_{k, \varepsilon}}(x)}{\left|\tilde{A}_{k, \varepsilon}\right|} \\
= & \sum_{|l| \leq m} \sum_{k=-\infty}\left(\sum_{j=-\infty}^{k} \int_{\mathbb{R}^{n}}(f * \phi)(y) \Phi_{j}(y) y^{l} d y\right) \\
& +\sum_{|l| \leq m}\left\{\left(\sum_{j=-\infty}^{k}(x) \chi_{\tilde{A}_{k, \varepsilon}}(x)-\frac{\psi_{l}^{k+1}(x) \chi_{\tilde{A}_{k+1, \varepsilon}}(x)}{\left|\tilde{A}_{k+1, \varepsilon}\right|}\right)\right. \\
& \left.+\sum_{k=2}^{\infty}\left(\int_{\mathbb{R}^{n}}(f * \phi)(y) \Phi_{k}(y) y^{l} d y\right) \frac{\psi_{l}^{k}(x) \chi_{\tilde{A}_{k, \varepsilon}}(x)}{\left|\tilde{A}_{k, \varepsilon}\right|}\right\} \\
= & I(x)+\psi_{l}^{1}(x) \chi_{\tilde{A}_{1, \varepsilon}}(x) \\
&
\end{aligned}
$$

We first decompose $I(x)$. From $\sum_{j=-\infty}^{k} \int_{\mathbb{R}^{n}} \Phi_{j}(y) y^{l} d y \leq C 2^{k(n+|l|)}$, it is easy to deduce that

$$
\left|\sum_{j=-\infty}^{k} \int_{\mathbb{R}^{n}}(f * \phi)(y) \Phi_{j}(y) y^{l} d y\right| \chi_{B\left(0,2^{k+2}\right)}(x) \leq C 2^{k(n+|l|)} \tilde{G}(f)(x) \chi_{B\left(0,2^{k+2}\right)}(x) .
$$

In addition, we have that

$$
\left|\frac{\psi_{l}^{k}(x) \chi_{\tilde{A}_{k, \varepsilon}}(x)}{\left|\tilde{A}_{k, \varepsilon}\right|}-\frac{\psi_{l}^{k+1}(x) \chi_{\tilde{A}_{k+1, \varepsilon}}(x)}{\tilde{A}_{k+1, \varepsilon}}\right| \leq C 2^{-k(n+|l|)} \sum_{j=k-1}^{k+1} \chi_{j}(x) .
$$


Set

$h_{k, l}^{1}(x)=\left(\sum_{j=-\infty}^{k} \int_{\mathbb{R}^{n}}(f * \phi)(y) \Phi_{j}(y) y^{l} d y\right)\left(\frac{\psi_{l}^{k}(x) \chi_{\tilde{A}_{k, \varepsilon}}(x)}{\left|\tilde{A}_{k, \varepsilon}\right|}-\frac{\psi_{l}^{k+1}(x) \chi_{\tilde{A}_{k+1, \varepsilon}}(x)}{\left|\tilde{A}_{k+1, \varepsilon}\right|}\right)$

and

$$
a_{k, l}^{1}=2^{-(k+2) \alpha}\left\|h_{k, l}\right\|_{L^{q(\cdot)}\left(\mathbb{R}^{n}\right)}^{-1} .
$$

Then $a_{k, l}^{1}$ is a central $(\alpha, q(\cdot))$-atom with support $B_{k+2}$.

Let $\tau_{k, l}^{1}=2^{(k+2) \alpha}\left\|h_{k, l}\right\|_{L^{q(\cdot)}\left(\mathbb{R}^{n}\right)}$. Then we have that

$$
I(x)=\sum_{|l| \leq m} \sum_{k=-\infty}^{0} \tau_{k, l}^{1} a_{k, l}^{1}(x)
$$

and

$$
\begin{aligned}
\sum_{|l| \leq m} \sum_{k=-\infty}^{0}\left|\tau_{k, l}^{1}\right|^{p} & \leq C \sum_{|l| \leq m} \sum_{k=-\infty}^{0} 2^{(k+2) \alpha p}\left(\sum_{j=k-1}^{k+2}\left\|\tilde{G}_{N}(f) \chi_{j}\right\|_{L^{q(\cdot)}\left(\mathbb{R}^{n}\right)}\right)^{p} \\
& \leq C \sum_{|l| \leq m} \sum_{k=-\infty}^{2} 2^{(k+2) \alpha p}\left\|\tilde{G}_{N}(f) \chi_{j}\right\|_{L^{q(\cdot)}\left(\mathbb{R}^{n}\right)}^{p} \\
& \leq C\|f\|_{h \dot{K}_{q(\cdot)}^{\alpha, p}\left(\mathbb{R}^{n}\right)^{p}}^{p}
\end{aligned}
$$

Now we decompose $J$. A trivial computation as above gives us that

$$
\left|\sum_{j=-\infty}^{1} \int_{\mathbb{R}^{n}}(f * \phi)(y) \Phi_{j}(y) y^{l} d y\right| \chi_{B_{2}}(x) \leq C \tilde{G}_{N}(f)(x) \chi_{B_{2}}(x) .
$$

Set

$$
h_{1, l}^{2}(x)=\sum_{j=-\infty}^{1}\left(\int_{\mathbb{R}^{n}}(f * \phi)(y) \Phi_{j}(y) y^{l} d y\right) \frac{\psi_{l}^{1}(x) \chi_{\tilde{A}_{1, \varepsilon}}(x)}{\left|\tilde{A}_{1, \varepsilon}\right|}
$$

and

We can verify that

$$
\lambda_{1, l}^{2}(x)=2^{\alpha}\left\|h_{1, l}^{2}\right\|_{L^{q(\cdot)\left(\mathbb{R}^{n}\right)}}
$$

$$
\left\|h_{1, l}^{2}\right\|_{L^{q(\cdot)\left(\mathbb{R}^{n}\right)}} \leq C \sum_{j=0}^{2}\left\|\tilde{G}_{N}(f) \chi_{j}\right\|_{L^{q(\cdot)\left(\mathbb{R}^{n}\right)}} .
$$

Moreover, $a_{1, l}^{2}(x)=\left(\lambda_{1, l}^{2}\right)^{-1} h_{1, l}^{2}(x)$ is a central $(\alpha, q(\cdot))$-block supported on $B_{2}$ and $h_{1, l}^{2}(x)=\left(\lambda_{1, l}^{2}\right) a_{1, l}^{2}(x)$. For integer $k \geq 2$, let

$$
h_{k, l}^{2}(x)=\left(\int_{\mathbb{R}^{n}}(f * \phi)(y) \Phi_{k}(y) y^{l} d y\right) \frac{\psi_{l}^{k}(x) \chi_{\tilde{A}_{k, \varepsilon}}(x)}{\left|\tilde{A}_{k, \varepsilon}\right|} .
$$

By Lemma 1.3 and the generalized Hölder inequality we have

$$
\begin{aligned}
\left\|h_{k, l}^{2}\right\|_{L^{q(\cdot)}\left(\mathbb{R}^{n}\right)} & \leq C\left\|\tilde{G}_{N}(f) \chi_{\tilde{A}_{k, \varepsilon}}\right\|_{L^{q(\cdot)}\left(\mathbb{R}^{n}\right)} \\
& \leq C \sum_{j=k-1}^{k+1}\left\|\tilde{G}_{N}(f) \chi_{A_{j}}\right\|_{L^{q(\cdot)}\left(\mathbb{R}^{n}\right)} .
\end{aligned}
$$


This implies that $a_{k, l}^{2}(x)=\left(\left\|h_{k, l}^{2}\right\|_{L^{q(\cdot)}\left(\mathbb{R}^{n}\right)} 2^{k \alpha}\right)^{-1} h_{k, l}^{2}(x)$ is a central $(\alpha, q(\cdot))$-block which is supported on $B_{k+1}$. Set $\lambda_{k, l}^{2}=\left\|h_{k, l}^{2}\right\|_{L^{q(\cdot)}\left(\mathbb{R}^{n}\right)} 2^{k \alpha}$. It then follows that

$$
J(x)=\sum_{|l| \leq m} \sum_{k=1}^{\infty} \lambda_{k, l}^{2} a_{k, l}^{2}(x)
$$

and

$$
\sum_{|l| \leq m} \sum_{k=1}^{\infty}\left|\lambda_{k, l}^{2}\right|^{p} \leq C \sum_{k=0}^{\infty} 2^{k \alpha p}\left\|\tilde{G}_{N}(f) \chi_{k}\right\|_{L^{q(\cdot)}\left(\mathbb{R}^{n}\right)}^{p} \leq C\|f\|_{h \dot{K}_{q(\cdot)}^{\alpha, p}\left(\mathbb{R}^{n}\right)}^{p} .
$$

Similar to the method of [10, Theorem 2.1], we can get

$$
f * \phi=\sum_{k=1}^{\infty} \lambda_{k} a_{k} \quad \text { in the sense of } \mathcal{S}^{\prime}\left(\mathbb{R}^{n}\right) .
$$

Now we prove the sufficiency. Let $\left\{\lambda_{k}\right\}_{k=-\infty}^{\infty}$ be a sequence of number such that $\sum_{k=-\infty}^{\infty}\left|\lambda_{k}\right|^{p}<\infty,\left\{a_{k}\right\}_{k \leq 0}$ be a sequence of central $(\alpha, q(\cdot))$-atom supported on $B_{k}$ and $\left\{a_{k}\right\}_{k>0}$ be a sequence of central $(\alpha, q(\cdot))$-block, and

$$
f=\sum_{k=-\infty}^{\infty} \lambda_{k} a_{k} \quad \text { in the sense of } \mathcal{S}^{\prime}\left(\mathbb{R}^{n}\right)
$$

Let $\phi \in \mathcal{S}\left(\mathbb{R}^{n}\right)$ such that $\int_{\mathbb{R}^{n}} \phi(x) d x=1, \quad \operatorname{supp} \phi \subset B(0,1)$. Our goal is to prove that

$$
\tilde{\phi}_{+}^{*}(f) \in \dot{K}_{q(\cdot)}^{\alpha, p}\left(\mathbb{R}^{n}\right) .
$$

To this aim, we consider the following two cases.

When $0<p \leq 1$. In this case, we just need to show that there exists a constant $C$ such that for any $a_{k}$,

$$
\left\|\tilde{\phi}_{+}^{*}\left(a_{k}\right)\right\|_{\dot{K}_{q(\cdot)}^{\alpha, p}\left(\mathbb{R}^{n}\right)} \leq C .
$$

If $k \leq 0$, write

$$
\begin{aligned}
\left\|\tilde{\phi}_{+}^{*}\left(a_{k}\right)\right\|_{\dot{K}_{q(\cdot)}^{\alpha, p}\left(\mathbb{R}^{n}\right)}^{p} & =\sum_{j=-\infty}^{k+3} 2^{j \alpha p}\left\|\tilde{\phi}_{+}^{*}\left(a_{k}\right) \chi_{A_{j}}\right\|_{L^{q(\cdot)}\left(\mathbb{R}^{n}\right)}^{p}+\sum_{j=k+4}^{\infty} 2^{j \alpha p}\left\|\tilde{\phi}_{+}^{*}\left(a_{k}\right) \chi_{A_{j}}\right\|_{L^{q(\cdot)}\left(\mathbb{R}^{n}\right)}^{p} \\
& =L_{1}^{1}+L_{2}^{1} .
\end{aligned}
$$

Applying the trivial estimate that

$$
\tilde{\phi}_{+}^{*}\left(a_{k}\right)(x) \leq C \mathcal{M} a_{k}(x)
$$

and the $L^{q(\cdot)}\left(\mathbb{R}^{n}\right)$-boundedness of $\mathcal{M}$, we see that

$$
L_{1}^{1} \leq \sum_{j=-\infty}^{k+3} 2^{j \alpha p}\left\|\mathcal{M} a_{k}\right\|_{L^{q(\cdot)}\left(\mathbb{R}^{n}\right)}^{p} \leq C \sum_{j=-\infty}^{k+3} 2^{j \alpha p}\left\|a_{k}\right\|_{L^{q(\cdot)}\left(\mathbb{R}^{n}\right)}^{p} \leq C .
$$


Now let $m \in \mathbb{Z}_{+}$such that $\alpha-n \delta_{2}<m+1$, and $\mathcal{J}_{m}$ be the $m$-order Taylor expansion for $\phi$ at $x / t$. For $x \in A_{j}$ with $j \geq k+4$, a straightforward computation gives us that

$$
\begin{aligned}
\left|a_{k} * \phi_{t}(x)\right| & \left.=t^{-n} \mid \int_{\mathbb{R}^{n}} a_{k}(y) \phi\left(\frac{x-y}{t}\right)\right) d y \mid \\
& =t^{-n}\left|\int_{\mathbb{R}^{n}} a_{k}(y)\left(\phi\left(\frac{x-y}{t}\right)-\mathcal{J}_{m}\left(\frac{y}{t}\right)\right) d y\right| \\
& \leq C \int_{\mathbb{R}^{n}}\left|a_{k}(y)\right||y|^{m+1}(t+|x-\theta y|)^{-(n+m+1)} d y \\
& \leq C \frac{2^{k(m+1)}}{|x|^{n+m+1}} \int_{\mathbb{R}^{n}}\left|a_{k}(y)\right| d y
\end{aligned}
$$

where $0<\theta<1$. Therefore, for $x \in A_{j}$ with $j \geq k+4$, we have

$$
\tilde{\phi}_{+}^{*}\left(a_{k}\right)(x) \leq C 2^{k(m+1)}|x|^{-(n+m+1)}\left|B_{k}\right|^{-\alpha / n}\left\|\chi_{B_{k}}\right\|_{L^{q^{\prime}(\cdot)}\left(\mathbb{R}^{n}\right)} .
$$

So by Lemma 1.2 and Lemma 1.3 we have

$$
\begin{aligned}
L_{2}^{1} & \leq C \sum_{j=k+4}^{\infty} 2^{p[k(m+1)-j(n+m+1)]}\left(\frac{\left|B_{j}\right|}{\left|B_{k}\right|}\right)^{p \alpha / n}\left\|\chi_{B_{k}}\right\|_{L^{q^{\prime}(\cdot)\left(\mathbb{R}^{n}\right)}}^{p}\left\|\chi_{B_{j}}\right\|_{L^{q(\cdot)}\left(\mathbb{R}^{n}\right)}^{p} \\
& \leq C \sum_{j=k+4}^{\infty} 2^{p[k(m+1)-j(n+m+1)]}\left(\frac{\left|B_{j}\right|}{\left|B_{k}\right|}\right)^{p \alpha / n}\left\|\chi_{B_{k}}\right\|_{L^{q^{\prime}(\cdot)\left(\mathbb{R}^{n}\right)}}^{p}\left(\left|B_{j}\right|\left\|_{B_{j}}\right\|_{L^{q^{\prime}(\cdot)\left(\mathbb{R}^{n}\right)}}^{-1}\right)^{p} \\
& =C \sum_{j=k+4}^{\infty} 2^{p(k-j)(m+1-\alpha)}\left(\frac{\left\|\chi_{B_{k}}\right\|_{L^{q^{\prime}(\cdot)}\left(\mathbb{R}^{n}\right)}}{\left\|\chi_{B_{j}}\right\|_{L^{q^{\prime}(\cdot)}\left(\mathbb{R}^{n}\right)}}\right)^{p} \\
& \leq C \sum_{j=k+4}^{\infty} 2^{p(k-j)\left(m+1-\alpha+n \delta_{2}\right)} \leq C .
\end{aligned}
$$

If $k \geq 1$, by $(2.1)$, the $L^{q(\cdot)}\left(\mathbb{R}^{n}\right)$-boundedness of $\mathcal{M}$ and $\operatorname{supp} \tilde{\phi}_{+}^{*}\left(a_{k}\right) \subset B_{k+1}$, we have

$$
\begin{aligned}
\left\|\tilde{\phi}_{+}^{*}\left(a_{k}\right)\right\|_{\dot{K}_{q(\cdot)}^{\alpha, p}\left(\mathbb{R}^{n}\right)}^{p} & =\sum_{j=-\infty}^{k+1} 2^{j \alpha p}\left\|\tilde{\phi}_{+}^{*}\left(a_{k}\right) \chi_{A_{j}}\right\|_{L^{q(\cdot)}\left(\mathbb{R}^{n}\right)}^{p} \\
& \leq C \sum_{j=-\infty}^{k+1} 2^{j \alpha p}\left\|M a_{k}\right\|_{L^{q(\cdot)}\left(\mathbb{R}^{n}\right)}^{p} \\
& \leq C \sum_{j=-\infty}^{k+1} 2^{(j-k) \alpha p} \leq C
\end{aligned}
$$


When $1<p<\infty$, take $1 / p+1 / p^{\prime}=1$. Write

$$
\begin{aligned}
\left\|\tilde{\phi}_{+}^{*}(f)\right\|_{\dot{K}_{q(\cdot)}^{\alpha, p}\left(\mathbb{R}^{n}\right)}^{p}= & \sum_{k=-\infty}^{\infty} 2^{\alpha k p}\left\|\tilde{\phi}_{+}^{*}(f) \chi_{k}\right\|_{L^{q(\cdot)}\left(\mathbb{R}^{n}\right)}^{p} \\
\leq & \sum_{k=-\infty}^{\infty} 2^{\alpha k p}\left(\sum_{j=-\infty}^{\infty}\left|\lambda_{j}\right|\left\|\tilde{\phi}_{+}^{*}\left(a_{j}\right) \chi_{k}\right\|_{L^{q(\cdot)}\left(\mathbb{R}^{n}\right)}\right)^{p} \\
\leq & C \sum_{k=-\infty}^{\infty} 2^{\alpha k p}\left(\sum_{j=-\infty}^{0}\left|\lambda_{j}\right|\left\|\tilde{\phi}_{+}^{*}\left(a_{j}\right) \chi_{k}\right\|_{L^{q(\cdot)}\left(\mathbb{R}^{n}\right)}\right)^{p} \\
& +C \sum_{k=-\infty}^{0} 2^{\alpha k p}\left(\sum_{j=1}^{\infty}\left|\lambda_{j}\right|\left\|\tilde{\phi}_{+}^{*}\left(a_{j}\right) \chi_{k}\right\|_{L^{q(\cdot)}\left(\mathbb{R}^{n}\right)}\right)^{p} \\
& +C \sum_{k=1}^{\infty} 2^{\alpha k p}\left(\sum_{j=1}^{\infty}\left|\lambda_{j}\right|\left\|\tilde{\phi}_{+}^{*}\left(a_{j}\right) \chi_{k}\right\|_{L^{q(\cdot)}\left(\mathbb{R}^{n}\right)}\right)^{p} \\
= & C\left(U_{1}+U_{2}+U_{3}\right) .
\end{aligned}
$$

For the term $U_{3}$, it follows from the Hölder inequality that

$$
\begin{aligned}
U_{3} & =\sum_{k=1}^{\infty} 2^{\alpha k p}\left(\sum_{j=k-1}^{\infty}\left|\lambda_{j}\right|\left\|\tilde{\phi}_{+}^{*}\left(a_{j}\right) \chi_{k}\right\|_{L^{q(\cdot)}\left(\mathbb{R}^{n}\right)}\right)^{p} \\
& \leq C \sum_{k=1}^{\infty} 2^{\alpha k p}\left(\sum_{j=k-1}^{\infty}\left|\lambda_{j}\right| 2^{-\alpha j}\right)^{p} \\
& \leq C \sum_{k=1}^{\infty} 2^{\alpha k p}\left(\sum_{j=k-1}^{\infty}\left|\lambda_{j}\right|^{p} 2^{-\alpha p j / 2}\right)\left(\sum_{j=k-1}^{\infty} 2^{-\alpha p^{\prime} j / 2}\right)^{p / p^{\prime}} \\
& \leq C \sum_{j=0}^{\infty}\left|\lambda_{j}\right|^{p}<\infty .
\end{aligned}
$$

On the other hand, a straightforward computation leads to that

$$
\begin{aligned}
U_{2} & =\sum_{k=-\infty}^{0} 2^{\alpha k p}\left(\sum_{j=1}^{\infty}\left|\lambda_{j}\right|\left\|\tilde{\phi}_{+}^{*}\left(a_{j}\right) \chi_{k}\right\|_{L^{q(\cdot)}\left(\mathbb{R}^{n}\right)}\right)^{p} \\
& \leq C \sum_{k=-\infty}^{0} 2^{\alpha k p}\left(\sum_{j=1}^{\infty}\left|\lambda_{j}\right| 2^{-\alpha j}\right)^{p} \\
& \leq C \sum_{j=1}^{\infty}\left|\lambda_{j}\right|^{p} \sum_{k=-\infty}^{0} 2^{\alpha k p}\left(\sum_{j=1}^{\infty} 2^{-\alpha p^{\prime} j}\right)^{p / p^{\prime}} \\
& \leq C \sum_{j=0}^{\infty}\left|\lambda_{j}\right|^{p}<\infty
\end{aligned}
$$


To estimate $U_{1}$, write

$$
\begin{aligned}
U_{1}= & \sum_{k=2}^{\infty} 2^{\alpha k p}\left(\sum_{j=-\infty}^{0}\left|\lambda_{j}\right|\left\|\tilde{\phi}_{+}^{*}\left(a_{j}\right) \chi_{k}\right\|_{L^{q(\cdot)}\left(\mathbb{R}^{n}\right)}\right)^{p} \\
& +\sum_{k=-\infty}^{1} 2^{\alpha k p}\left(\sum_{j=-\infty}^{k-2}\left|\lambda_{j}\right|\left\|\tilde{\phi}_{+}^{*}\left(a_{j}\right) \chi_{k}\right\|_{L^{q(\cdot)}\left(\mathbb{R}^{n}\right)}\right)^{p} \\
& +\sum_{k=-\infty}^{1} 2^{\alpha k p}\left(\sum_{j=k-1}^{0}\left|\lambda_{j}\right|\left\|\tilde{\phi}_{+}^{*}\left(a_{j}\right) \chi_{k}\right\|_{L^{q(\cdot)}\left(\mathbb{R}^{n}\right)}\right)^{p} \\
= & U_{1}^{1}+U_{1}^{2}+U_{1}^{3} .
\end{aligned}
$$

The Hölder inequality, along with the estimate (2.1), gives us that

$$
\begin{aligned}
U_{1}^{3} & \leq C \sum_{k=-\infty}^{1} 2^{\alpha k p}\left(\sum_{j=k-1}^{0}\left|\lambda_{j}\right|\left\|a_{j}\right\|_{L^{q(\cdot)}\left(\mathbb{R}^{n}\right)}\right)^{p} \\
& \leq C \sum_{k=-\infty}^{1} 2^{\alpha k p}\left(\sum_{j=k-1}^{0}\left|\lambda_{j}\right| 2^{-\alpha j}\right)^{p} \\
& \leq C \sum_{j=-\infty}^{0}\left|\lambda_{j}\right|^{p}<\infty .
\end{aligned}
$$

On the other hand, for $x \in A_{k}$ with $k \geq j+2$ and $k \leq 1$, similar to the first case we have

$$
\tilde{\phi}_{+}^{*}\left(a_{j}\right)(x) \leq C 2^{j(m+1)}|x|^{-(n+m+1)}\left|B_{j}\right|^{-\alpha / n}\left\|\chi_{B_{j}}\right\|_{L^{q^{\prime}(\cdot)\left(\mathbb{R}^{n}\right)}} .
$$

Note that $\alpha-n \delta_{2}<m+1$, so we have

$$
\begin{aligned}
& U_{1}^{2} \\
& \leq C \sum_{k=-\infty}^{1}\left(\sum_{j=-\infty}^{k-2}\left|\lambda_{j}\right| 2^{j(m+1)-k(n+m+1)}\left(\frac{\left|B_{k}\right|}{\left|B_{j}\right|}\right)^{\alpha / n}\left\|\chi_{B_{j}}\right\|_{L^{q^{\prime}(\cdot)}\left(\mathbb{R}^{n}\right)}\left\|\chi_{B_{k}}\right\|_{L^{q(\cdot)}\left(\mathbb{R}^{n}\right)}\right)^{p} \\
& \leq C \sum_{k=-\infty}^{1}\left(\sum_{j=-\infty}^{k-2}\left|\lambda_{j}\right| 2^{(j-k)\left(m+1-\alpha+n \delta_{2}\right)}\right)^{p} \\
& \leq C \sum_{j=-\infty}^{-1}\left|\lambda_{j}\right|^{p}<\infty .
\end{aligned}
$$

Similarly, we have

$$
\begin{aligned}
U_{1}^{1} & \leq C \sum_{k=2}^{\infty}\left(\sum_{j=-\infty}^{0}\left|\lambda_{j}\right| 2^{(j-k)\left(m+1-\alpha+n \delta_{2}\right)}\right)^{p} \\
& \leq C \sum_{j=-\infty}^{0}\left|\lambda_{j}\right|^{p}<\infty
\end{aligned}
$$


Combining the estimates above, it will show that if $f$ has the decomposition $f=\sum_{j=-\infty}^{\infty} \lambda_{j} a_{j}$, then

$$
\left\|\tilde{\phi}_{+}^{*}(f)\right\|_{\dot{K}_{q(\cdot)}^{\alpha, p}\left(\mathbb{R}^{n}\right)}^{p}<\infty .
$$

This completes the proof of Theorem 2.4.

Similar to the proof of Theorem 2.4, we are easy to get the following conclusion, which gives another characterization of $h K_{q(\cdot)}^{\alpha, p}\left(\mathbb{R}^{n}\right)$.

Theorem 2.5. Let $n \delta_{2} \leq \alpha<\infty, 0<p<\infty$ and $q(\cdot) \in \mathcal{B}\left(\mathbb{R}^{n}\right)$. Then $f \in$ $h K_{q(\cdot)}^{\alpha, p}\left(\mathbb{R}^{n}\right)$ if and only if $f \in K_{q(\cdot)}^{\alpha, p}\left(\mathbb{R}^{n}\right)$. That is,

$$
f(x)=\sum_{k=0}^{\infty} \lambda_{k} a_{k}(x), \quad \text { in the sense of } \mathcal{S}^{\prime}\left(\mathbb{R}^{n}\right),
$$

where each $a_{k}$ is a central $(\alpha, q(\cdot))$-block of restricted type, and $\left\{\lambda_{k}\right\}_{k=0}^{\infty}$ satisfies that $\sum_{k=0}^{\infty}\left|\lambda_{k}\right|^{p}<\infty$. Moreover,

$$
\|f\|_{h K_{q(\cdot)}^{\alpha, p}\left(\mathbb{R}^{n}\right)} \approx \inf \left(\sum_{k=0}^{\infty}\left|\lambda_{k}\right|^{p}\right)^{1 / p},
$$

where the infimum is taken over all above decomposition of $f$.

The boundedness of pseudo-differential operators on Herz-type spaces was studied by many authors (see [3, 8]). In the following part, as an application of Theorem 2.4, we will prove the boundedness of pseudo-differential operators of order zero on the space $h \dot{K}_{q(\cdot)}^{\alpha, p}\left(\mathbb{R}^{n}\right)$, which generalizes the result in [3].

Theorem 2.6. Let $n \delta_{2} \leq \alpha<\infty, 0<p<\infty$ and $q(\cdot) \in \mathcal{B}\left(\mathbb{R}^{n}\right)$. If $\operatorname{Tf}(x)=$ $\int_{\mathbb{R}^{n}} \hat{f}(x) \sigma(x, \xi) e^{2 \pi i x \cdot \xi} d \xi$ with $\sigma \in \mathbb{S}^{0}$, that is, $\sigma \in \mathcal{C}^{\infty}\left(\mathbb{R}^{n} \times \mathbb{R}^{n}\right)$ and $\left|D_{x}^{\alpha} D_{\xi}^{\beta} \sigma(x, \xi)\right| \leq$ $C_{\alpha, \beta}(1+|\xi|)^{-|\beta|}$, then $\|T f\|_{h \dot{K}_{q(\cdot)}^{\alpha, p}\left(\mathbb{R}^{n}\right)} \leq C\|f\|_{h \dot{K}_{q(\cdot)}^{\alpha, p}\left(\mathbb{R}^{n}\right)}$.

Proof. Let $f \in h \dot{K}_{q(\cdot)}^{\alpha, p}\left(\mathbb{R}^{n}\right)$. By Theorem 2.4, we have $f(x)=\sum_{k=-\infty}^{\infty} \lambda_{k} a_{k}(x)$ in distributional sense. Then we consider two cases with $0<p \leq 1$ and $1<p<\infty$.

When $0<p \leq 1$. In this case, we only need to show that $\left\|T a_{k}\right\|_{h \dot{K}_{q(\cdot)}^{\alpha, p}\left(\mathbb{R}^{n}\right)} \leq C$ and $C$ is independent of $a_{k}$. If $k \leq 0$, then

$$
\begin{aligned}
\left\|T a_{k}\right\|_{h \dot{K}_{q(\cdot)}^{\alpha, p}\left(\mathbb{R}^{n}\right)}^{p} & =\sum_{j=-\infty}^{\infty} 2^{j \alpha p}\left\|\tilde{G}_{N}\left(T a_{k}\right) \chi_{j}\right\|_{L^{q(\cdot)}\left(\mathbb{R}^{n}\right)}^{p} \\
& =\sum_{j=-\infty}^{k+2} 2^{j \alpha p}\left\|\tilde{G}_{N}\left(T a_{k}\right) \chi_{j}\right\|_{L^{q(\cdot)\left(\mathbb{R}^{n}\right)}}^{p}+\sum_{j=k+3}^{\infty} 2^{j \alpha p}\left\|\tilde{G}_{N}\left(T a_{k}\right) \chi_{j}\right\|_{L^{q(\cdot)}\left(\mathbb{R}^{n}\right)}^{p} \\
& =I_{1}+I_{2} .
\end{aligned}
$$


For $I_{1}$, using the $L^{q(\cdot)}\left(\mathbb{R}^{n}\right)$-boundedness of $\mathcal{M}$, we have

$$
\begin{aligned}
I_{1} & =\sum_{j=-\infty}^{k+2} 2^{j \alpha p}\left\|\tilde{G}_{N}\left(T a_{k}\right) \chi_{j}\right\|_{L^{q(\cdot)}\left(\mathbb{R}^{n}\right)}^{p} \\
& \leq C \sum_{j=-\infty}^{k+2} 2^{j \alpha p}\left\|\mathcal{M}\left(T a_{k}\right)\right\|_{L^{q(\cdot)}\left(\mathbb{R}^{n}\right)}^{p} \\
& \leq C \sum_{j=-\infty}^{k+2} 2^{j \alpha p}\left\|a_{k}\right\|_{L^{q(\cdot)}\left(\mathbb{R}^{n}\right)}^{p} \\
& \leq C \sum_{j=-\infty}^{k+2} 2^{(j-k) \alpha p} \leq C .
\end{aligned}
$$

To estimate $I_{2}$, by Theorem 4 in [4], we can write that

$$
\phi_{t} *\left(T a_{k}\right)(x)=\int_{\mathbb{R}^{n}} K_{t}(x, x-z) a_{k}(z) d z .
$$

Then we expand $K_{t}(x, x-z)$ in a Taylor series about $z=0$. By the vanishing moments of $a_{k}$, we get that

$$
\phi_{t} *\left(T a_{k}\right)(x)=\sum_{|\alpha|=N+1} \int_{\mathbb{R}^{n}} D_{z}^{\alpha} K_{t}(x, x-\theta z) z^{\alpha} a_{k}(z) d z,
$$

where $\theta \in(0,1)$ and $N \in \mathbb{Z}_{+}$satisfying that $\alpha-n \delta_{2}<N+1$. Noting that $x \in A_{j}$ with $j \geq k+3$, by Theorem 4 in [4], we can obtain that

$$
\begin{aligned}
\left|\phi_{t} *\left(T a_{k}\right)(x)\right| & \leq \frac{C}{|x|^{n+N+1}} \int_{\mathbb{R}^{n}}|z|^{N+1} a_{k}(z) d z \\
& \leq \frac{C 2^{k(N+1)}}{|x|^{n+N+1}} \int_{\mathbb{R}^{n}} a_{k}(z) d z \\
& \leq \frac{C 2^{k(N+1)}}{|x|^{n+N+1}}\left\|a_{k}\right\|_{L^{q(\cdot)}\left(\mathbb{R}^{n}\right)}\left\|\chi_{B_{k}}\right\|_{L^{q^{\prime}(\cdot)\left(\mathbb{R}^{n}\right)}} \\
& \leq \frac{C 2^{k(N+1)}}{2^{j(n+N+1)}}\left|B_{k}\right|^{-\alpha / n}\left\|\chi_{B_{k}}\right\|_{L^{q^{(} \cdot(\cdot)\left(\mathbb{R}^{n}\right)}} .
\end{aligned}
$$

So by Lemma 1.2 and Lemma 1.3 we have

$$
\begin{aligned}
I_{2} & \leq C \sum_{j=k+3}^{\infty} 2^{p[k(N+1)-j(n+N+1)]}\left(\frac{\left|B_{j}\right|}{\left|B_{k}\right|}\right)^{p \alpha / n}\left\|\chi_{B_{k}}\right\|_{L^{q^{\prime}(\cdot)}\left(\mathbb{R}^{n}\right)}^{p}\left\|\chi_{B_{j}}\right\|_{L^{q(\cdot)}\left(\mathbb{R}^{n}\right)}^{p} \\
& \leq C \sum_{j=k+3}^{\infty} 2^{p[(k-j)(N+1)-j n]}\left(\frac{\left|B_{j}\right|}{\left|B_{k}\right|}\right)^{p \alpha / n}\left\|\chi_{B_{k}}\right\|_{L^{q^{\prime}(\cdot)\left(\mathbb{R}^{n}\right)}}^{p}\left(\left|B_{j}\right|\left\|\chi_{B_{j}}\right\|_{L^{q^{\prime}(\cdot)\left(\mathbb{R}^{n}\right)}}^{-1}\right)^{p} \\
& =C \sum_{j=k+3}^{\infty} 2^{p(k-j)(N+1-\alpha)}\left(\frac{\left\|\chi_{B_{k}}\right\|_{L^{q^{\prime}(\cdot)}\left(\mathbb{R}^{n}\right)}}{\left\|\chi_{B_{j}}\right\|_{L^{q^{\prime}(\cdot)\left(\mathbb{R}^{n}\right)}}}\right)^{p} \\
& \leq C \sum_{j=k+3}^{\infty} 2^{p(k-j)\left(N+1-\alpha+n \delta_{2}\right)} \leq C .
\end{aligned}
$$


If $k>0$, we choose a radial smooth function $\eta$ such that $\operatorname{supp} \eta \subset B(0,1)$ and $\eta$ equals 1 near the origin. We split $T=T_{1}+T_{2}$ by decomposing $K(x, z)=$ $K_{1}(x, z)+K_{2}(x, z)=\eta(z) K(x, z)+(1-\eta(z)) K(x, z)$. Then $T_{1}$ and $T_{2}$ are of order zero. Noting that $\operatorname{supp} \tilde{\phi}_{+}^{*}\left(T_{1} a_{k}\right) \subset B_{k+1}$ and $L^{q(\cdot)}\left(\mathbb{R}^{n}\right)$-boundedness of $\mathcal{M}$, we get that

$$
\begin{aligned}
\left\|T_{1} a_{k}\right\|_{h \dot{K}_{q(\cdot)}^{\alpha, p}\left(\mathbb{R}^{n}\right)}^{p} & =\sum_{j=-\infty}^{k+1} 2^{j \alpha p}\left\|\tilde{\phi}_{+}^{*}\left(T_{1} a_{k}\right) \chi_{j}\right\|_{L^{q(\cdot)}\left(\mathbb{R}^{n}\right)}^{p} \\
& \leq C \sum_{j=-\infty}^{k+1} 2^{j \alpha p}\left\|\mathcal{M}\left(T_{1} a_{k}\right)\right\|_{L^{q(\cdot)}\left(\mathbb{R}^{n}\right)}^{p} \\
& \leq C \sum_{j=-\infty}^{k+1} 2^{j \alpha p}\left\|T_{1} a_{k}\right\|_{L^{q(\cdot)}\left(\mathbb{R}^{n}\right)}^{p} \\
& \leq C \sum_{j=-\infty}^{k+1} 2^{j \alpha p}\left\|a_{k}\right\|_{L^{q(\cdot)}\left(\mathbb{R}^{n}\right)}^{p} \\
& \leq C \sum_{j=-\infty}^{k+1} 2^{(j-k) \alpha p} \leq C .
\end{aligned}
$$

To estimate $T_{2} a_{k}(x)$, we have

$$
\left|\left(K_{2}\right)_{t}(x, z)\right| \leq C_{M}(1+|z|)^{-M}
$$

for any $M \geq n$ (see [4, Theorem 4]). Then we write that

$$
\begin{aligned}
\left\|T_{2} a_{k}\right\|_{h \dot{K}_{q(\cdot)}^{\alpha, p}\left(\mathbb{R}^{n}\right)}^{p} & =\sum_{j=-\infty}^{\infty} 2^{j \alpha p}\left\|\tilde{\phi}_{+}^{*}\left(T_{2} a_{k}\right) \chi_{j}\right\|_{L^{q(\cdot)}\left(\mathbb{R}^{n}\right)}^{p} \\
& =\sum_{j=-\infty}^{k+2} 2^{j \alpha p}\left\|\tilde{\phi}_{+}^{*}\left(T_{2} a_{k}\right) \chi_{j}\right\|_{L^{q(\cdot)}\left(\mathbb{R}^{n}\right)}^{p}+\sum_{j=k+3}^{\infty} 2^{j \alpha p}\left\|\tilde{\phi}_{+}^{*}\left(T_{2} a_{k}\right) \chi_{j}\right\|_{L^{q(\cdot)}\left(\mathbb{R}^{n}\right)}^{p} \\
& =I I_{1}+I I_{2} .
\end{aligned}
$$

About $I I_{1}$, we can obtain the desirable estimate by a similar method to $I_{1}$. For $I I_{2}$, noting that $x \in A_{j}$ and $l \geq k+3$, by (2.2) we can obtain that

$$
\begin{aligned}
\left|\phi_{t} *\left(T_{2} a_{k}\right)(x)\right| & =\left|\int_{\mathbb{R}^{n}}\left(K_{2}\right)_{t}(x, x-z) a_{k}(z) d z\right| \\
& \leq C_{M} \int_{\mathbb{R}^{n}} \frac{1}{(1+|x-z|)^{M}}\left|a_{k}(z)\right| d z \\
& \leq \frac{C^{n}}{|x|^{n+N+1}} \int_{\mathbb{R}^{n}}\left|a_{k}(z)\right| d z \\
& \leq \frac{C 2^{k(N+1)}}{|x|^{n+N+1}}\left|B_{k}\right|^{-\alpha / n}\left\|\chi_{B_{k}}\right\|_{L^{q^{\prime}(\cdot)}\left(\mathbb{R}^{n}\right)}
\end{aligned}
$$

where we take $N \in \mathbb{Z}_{+}$satisfying that $\alpha-n \delta_{2}<N+1$. So it is readily to follow that $I I_{2} \leq C$. 
When $1<p<\infty$. In this case, we write that

$$
\begin{aligned}
\|T f\|_{h \dot{K}_{q(\cdot)}^{\alpha, p}\left(\mathbb{R}^{n}\right)} & =\left\{\sum_{k=-\infty}^{\infty} 2^{k \alpha p}\left\|\tilde{\phi}_{+}^{*}(T f) \chi_{k}\right\|_{L^{q(\cdot)}\left(\mathbb{R}^{n}\right)}^{p}\right\}^{1 / p} \\
\leq & \left\{\sum_{k=-\infty}^{\infty} 2^{k \alpha p}\left(\sum_{j=-\infty}^{\infty}\left|\lambda_{j}\right|\left\|\tilde{\phi}_{+}^{*}\left(T a_{j}\right) \chi_{k}\right\|_{L^{q(\cdot)}\left(\mathbb{R}^{n}\right)}\right)^{p}\right\}^{1 / p} \\
\leq & \left\{\sum_{k=-\infty}^{\infty} 2^{k \alpha p}\left(\sum_{j=-\infty}^{k-1}\left|\lambda_{j}\right|\left\|\tilde{\phi}_{+}^{*}\left(T a_{j}\right) \chi_{k}\right\|_{L^{q(\cdot)}\left(\mathbb{R}^{n}\right)}\right)^{p}\right\}^{1 / p} \\
& +\left\{\sum_{k=-\infty}^{\infty} 2^{k \alpha p}\left(\sum_{j=k}^{\infty}\left|\lambda_{j}\right|\left\|\tilde{\phi}_{+}^{*}\left(T a_{j}\right) \chi_{k}\right\|_{L^{q(\cdot)}\left(\mathbb{R}^{n}\right)}\right)^{p}\right\}^{1 / p} \\
= & I I I_{1}+I I I_{2} .
\end{aligned}
$$

Similar to $I_{1}$, we can get the estimates of $I I I_{2}$. For $I I I_{1}$, we continue to decompose it as follows.

$$
\begin{aligned}
I I I_{1}= & \left\{\sum_{k=-\infty}^{\infty} 2^{k \alpha p}\left(\sum_{j=-\infty}^{k-1}\left|\lambda_{j}\right|\left\|\tilde{\phi}_{+}^{*}\left(T a_{j}\right) \chi_{k}\right\|_{L^{q(\cdot)}\left(\mathbb{R}^{n}\right)}\right)^{p}\right\}^{1 / p} \\
\leq & \left\{\sum_{k=-\infty}^{0} 2^{k \alpha p}\left(\sum_{j=-\infty}^{k-1}\left|\lambda_{j}\right|\left\|\tilde{\phi}_{+}^{*}\left(T a_{j}\right) \chi_{k}\right\|_{L^{q(\cdot)}\left(\mathbb{R}^{n}\right)}\right)^{p}\right\}^{1 / p} \\
& +\left\{\sum_{k=1}^{\infty} 2^{k \alpha p}\left(\sum_{j=-\infty}^{k-1}\left|\lambda_{j}\right|\left\|\tilde{\phi}_{+}^{*}\left(T_{1} a_{j}\right) \chi_{k}\right\|_{L^{q(\cdot)}\left(\mathbb{R}^{n}\right)}\right)^{p}\right\}^{1 / p} \\
& +\left\{\sum_{k=1}^{\infty} 2^{k \alpha p}\left(\sum_{j=-\infty}^{k-1}\left|\lambda_{j}\right|\left\|\tilde{\phi}_{+}^{*}\left(T_{2} a_{j}\right) \chi_{k}\right\|_{L^{q(\cdot)}\left(\mathbb{R}^{n}\right)}\right)^{p}\right\}^{1 / p} \\
= & I I I_{1}^{1}+I I I_{1}^{2}+I I I_{1}^{3} .
\end{aligned}
$$

For $I I I_{1}^{2}$, it is easy to get the estimate by a similar method to $I_{1}$. Using the vanishing moments for $I I I_{1}^{1}$ and (2.2) for $I I I_{1}^{3}$, it is readily to follow that if $x \in A_{k}$ and $k \geq j+1$, then

$$
\left|\tilde{\phi}_{+}^{*}\left(T a_{j}\right)(x)\right|,\left|\tilde{\phi}_{+}^{*}\left(T_{2} a_{j}\right)(x)\right| \leq \frac{C 2^{j(N+1)}}{|x|^{n+N+1}}\left|B_{j}\right|^{-\alpha / n}\left\|\chi_{B_{j}}\right\|_{L^{q^{\prime}(\cdot)}\left(\mathbb{R}^{n}\right)},
$$

where we choose $N \in \mathbb{Z}_{+}$such that $\alpha-n \delta_{2}<N+1$. From this, it is readily to follow that $I I I_{1}^{1}+I I I_{1}^{3} \leq C$.

This completes the proof of Theorem 2.6.

Acknowledgement. The authors are very grateful to the referees for their valuable comments. This work was supported by the National Natural Science Foundation of China (Grant No. 11171345) and the Specialized Research Fund for the Doctoral Program of Higher Education of China (Grant No. 20120023110003). 


\section{REFERENCES}

1. D.V. Cruz-Uribe and A. Fiorenza, Variable Lebesgue Spaces: Foundations and Harmonic Analysis, Applied and Numerical Harmonic Analysis, Birkhäuser/Springer, Heidelberg, 2013.

2. L. Diening, P. Harjulehto, P. Hästö and M. Růžička, Lebesgue and Sobolev spaces with variable exponents, Lecture Notes in Math., vol. 2017, Springer, Heidelberg, 2011.

3. D. Fan and D. Yang, The weighted Herz-type Hardy spaces $h \dot{K}_{q}^{\alpha, p}\left(\omega_{1}, \omega_{2}\right)$, Approx. Theory Appl. (N.S.) 13 (1997), no. 4, 19-41.

4. D. Goldberg, A Local Version of Real Hardy Spaces, Duke Math. J. 46 (1979), 27-42.

5. M. Izuki, Boundedness of sublinear operators on Herz spaces with variable exponent and application to wavelet characterization, Anal. Math. 36 (2010), 33-50.

6. O. Kováčik and J. Rákosník, On spaces $L^{p(x)}$ and $W^{k, p(x)}$, Czechoslovak Math. J. 116 (1991), 592-618.

7. S. Lu and D. Yang, The local versions of $H^{p}\left(\mathbb{R}^{n}\right)$ spaces at the origin, Studia Math. 116 (1995), 103-131.

8. Y. Tsutsui, Pseudo-differential operators of class $S_{0,0}^{m}$ on the Herz-type spaces, Hokkaido Math. J. 38 (2009), 283-302.

9. H. Wang, The decomposition for the Herz space with variable exponent and its applications, 1425-1431, Proceedings of Academic Conference on Research Achievements of the Fundamental Research Funds for the Central Universities, China Coal Industry Publishing House, 2011.

10. H. Wang and Z. Liu, The Herz-type Hardy spaces with variable exponent and its applications, Taiwanese J. Math. 16 (2012), 1363-1389.

11. H. Wang and Z. Liu, Some characterizations of Herz-type Hardy spaces with variable exponent, Ann. Funct. Anal. 6 (2015), 224-233.

1 Department of Mathematical and Physical Science, Zibo Normal College, Zibo 255130, Shandong, P. R. China.

E-mail address: hbwang_2006@163.com

2 Department of Mathematics, China University of Mining and Technology(Beijing), BeiJing 100083, P. R. China.

E-mail address: liuzg@cumtb.edu.cn 